\title{
Automatic Generation of Extended UIO Sequences for Communication Protocols in an EFSM Model
}

\author{
Xiangdong Li, Teruo Higashino, Masahiro Higuchi and Kenichi Taniguchi \\ Department of Information and Computer Sciences, Osaka University \\ Machikaneyama 1-3, Toyonaka, Osaka 560, Japan \\ Tel: +81-6-850-6607 Fax: +81-6-850-6609 \\ E-mail : \{lidong, higashino, higuchi, taniguchi\}@ics.es.osaka-u.ac.jp
}

Keyword Codes : C.2.2, D.2.5, F.4.3

Keyword : Network Protocols, Testing and Debugging, Formal Languages

\begin{abstract}
In this paper, we propose an automatic test case derivation method for an extended finite state machine model called EFSM/pres where extended UIO sequences called E-UIO sequences are generated. In the EFSM/pres model, each EFSM has a finite control and a finite number of registers where the data types of $\mathrm{I} / \mathrm{O}$ data and registers' values are restricted to the integer type. The next registers' values are represented as linear combinations of the input values and current register values. The transition conditions on input variables and register variables are written using addition, subtraction, comparison and Boolean operators. For such a restricted class, we enumerate every transition sequence and decide whether the sequence is an E-UIO sequence for a given state mechanically. Concrete data values for the E-UIO sequence are also generated mechanically where a decision procedure for Presburger arithmetic is used. The derivation of E-UIO sequences for a part of OSI Session protocol is given as an example.
\end{abstract}

\section{Introduction}

Precise specifications are essential for the design and implementation of distributed systems and communication networks. They are important during the validation of the system design, implementation development and conformance testing phase. The use of formal description techniques (FDT's) allows the automation of conformance testing [BoUy 91]. In the protocol conformance testing, there are two aspects. One is the control flow. The other is the data flow. The control flow is usually described as a simple specification model of finite state machines (FSM's). While the test cases can be generated for the FSM models automatically, they become usually infeasible for more complex models, such as extended FSM's because of the data parameters. 
The selection of appropriate test cases including data parameters is an important issue for conformance testing of communication protocols, especially for practical purposes. Certain authors have considered extended finite state machine (EFSM) specifications which include data parameters and additional state variables. Usually, the data flow relations between input/output parameters and state variables are considered in the test selection process [Sari 87], however, it is generally assumed that the transitions do not contain enabling conditions depending on the additional state variables, or such dependencies are treated in an informal manner. The approach of [TrSa 89] considers the data flow relation automatically, while [Tret 89] leaves certain aspects to be carried out interactively, as certain problems can not be solved automatically in the general case. The approach of [ChZh 94] generates the values of the data parameters using a heuristic constraints solver automatically. In the approach of [UrYa 91], first, a flow graph is constructed. Then, the relations between input and output data are observed and the test cases are derived based on the observations. The approach of [WaLi 92a, WaLi 92b] proposes an axiomatic approach to generate test cases where, by traversing a given path in the EFSM carefully, the extended path is generated so that the derived path satisfies the constraints necessary for tracing the given path. Symbolic executors may be also used for test case generations for some cases. For example, a LOTOS tool SMILE has been developed for the symbolic execution of full LOTOS specifications [EeWo 92]. This tool can be used to generate test sequences using a narrowing technique for some cases. However, automatic test case generation is impossible in general.

In this paper, we introduce an extended finite state machine model called EFSM/pres (EFSM model with Presburger arithmetic) and propose a method to generate extended UIO sequences called E-UIO sequences. In the EFSM/pres model, each EFSM has a finite control and a finite number of registers. As the data types, we only consider Presburger arithmetic [HoUl 79], that is, we only consider the data parameters belonging to the integer type. The next registers' values must be represented as linear combinations of the input values and current register values. The operations used for representing transition conditions are restricted to addition, subtraction, comparison and Boolean operators. In general, in EFSM models, transition conditions may depend on the registers' values. Therefore, in order to execute a given UIO sequence, the registers' values satisfying all the transition conditions in the UIO sequence must be assigned at the starting state of the UIO sequence. Such values may be assigned by executing a specific preceding sequence before the UIO sequence is executed. Here, as an extended UIO sequence, we consider the transition sequence which is obtained by concatenating the preceding sequence and the UIO sequence and we call it an E-UIO sequence. In our restricted class, first, we find a transition sequence as a candidate using breadth first search and decide whether the sequence becomes an E-UIO sequence for a given state mechanically. Concrete data values for the E-UIO sequence are also generated mechanically where a decision procedure for Presburger arithmetic is used. Since, in general, it is undecidable whether there exists an E-UIO sequence for a given state, the proposed method generates an E-UIO sequence for a given state automatically only if it exists. 
In [HiBo 92, HiBo 94], we introduced a basic idea to generate test cases for a restricted class of LOTOS expressions with data parameters. In this paper, we generalize the method so that we can generate the E-UIO sequences from a given EFSM automatically if they exist. The paper is structured as follows. The definition of our EFSM/pres model is given in Section 2. The reason why the general testing methods using UIO sequences for FSM models cannot be applied for EFSM models is explained in Section 3. We also describe the problem that we must solve to generate extended UIO sequences for EFSM models automatically. In Section 4, we introduce the notion of E-UIO sequences and provide an idea to solve the problem. Using this method, we can generate the E-UIO sequences with data values automatically from a given EFSM. In Section 5, the derivation of E-UIO sequences for a part of OSI Session protocol is described as an example.

\section{Our EFSM Model}

In this paper, we use an extended finite state machine (EFSM) model called EFSM/pres. First, we introduce some notations necessary for defining the EFSM/pres model formally.

[Definition 2.1]

A term which consists of integers, variables of integer type, and operators " $+"$ and " $-"$ is called a $P$-term. A $P$-sentence is defined inductively as follows.

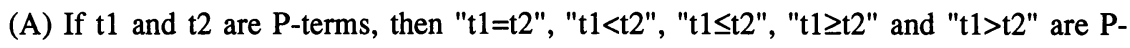
sentences.

(B) If $\alpha$ and $\beta$ are P-sentences, then "( $\alpha)$ and $(\beta)$ ", " $(\alpha)$ or $(\beta)$ ", "not $(\alpha)$ ", " $(\alpha) \supset(\beta)$ " are Psentences.

For example, " $x$ ", "3" and " $x+y-3$ " are P-terms. The expressions " $x=y+1$ " and "( $x \geq y-z)$ or $(\mathrm{z}=\mathrm{w})$ " are $\mathrm{P}$-sentences. However, " $\mathrm{x}^{2}+2 \mathrm{x}-3=0$ " is not a P-sentence because multiplication is used.

Using these notations, we define the EFSM/pres model formally. In our EFSM/pres model, an EFSM has a finite state control and a finite number of registers $R_{1}, \ldots, R_{n}$ where " $n$ " denotes the number of the registers. The specification of an EFSM is described as a labeled directed graph such as Fig. 1. The types of all registers must be integers. We assume that the number of $\mathrm{I} / \mathrm{O}$ gates is finite. Each node and edge represent a state of the finite control and a transition, respectively. At the initial state sinit, the initial values $R_{1 . \text { init }}, \ldots, R_{n . i n i t}$ of the registers are specified. Each edge has a label whose form is $\langle\mathrm{C}, \mathrm{a}$ ? $\mathrm{x} / \mathrm{b}$ !E,RD $>$. Here, "a" and " $\mathrm{b}$ " denote gate names. The symbol " $a$ ? $x$ " denotes an input event and the variable " $x$ " represents an input value given from the gate "a". More than one input values may be given for an input event. Such an input event is described like as "a?y,z". The event "b!E( $\left.x, R_{1}, \ldots, R_{n}\right)$ " denotes an output event and the value of the expression " $E\left(x, R_{1}, \ldots, R_{n}\right)$ " is emitted from the gate " $b$ " (more than one outputs may be emitted in an output event). The expression $E\left(x, R_{1}, \ldots, R_{n}\right)$ must be a P-term. A pair of one input event and one output event is executed at each transition. Such a pair is called an $\mathrm{I} / \mathrm{O}$ event. The first element " $\mathrm{C}$ " of the label is called a transition condition. A transition condition " $\mathrm{C}$ " in $<\mathrm{C}, \mathrm{a}$ ? $\mathrm{x} / \mathrm{b}$ !E, RD $>$ must be a P-sentence which may contain the variable " $x$ " and registers " $R_{1} ", \ldots,, R_{n}$ ". The value of the transition condition " $C$ " is decided by 
substituting the input and the current values of the registers into " $x$ " and " $R_{1} ", \ldots,{ }^{n}$ ", respectively. If the value of the transition condition "C" in $\langle C, a ? \mathrm{x} / \mathrm{b} ! \mathrm{E}, \mathrm{RD}\rangle$ is true, then the $\mathrm{I} / \mathrm{O}$ event " $\mathrm{a}$ ? $\mathrm{x} / \mathrm{b}$ !E" can be executed. Otherwise, it is not executable. We assume that the EFSM is deterministic. That is, if there are state transitions "s-<C,$a$ ? $x / b ! E_{1}, R_{1}>->t_{1}$ ", "s-

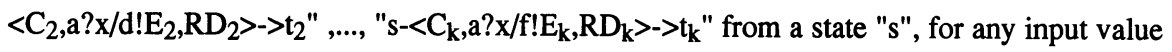
and any register values, at most one of $\mathrm{C}_{1}, \ldots, \mathrm{C}_{\mathrm{k}}$ must be true. The third element $\mathrm{RD}$ in the label $<\mathrm{C}, \mathrm{a}$ ? $\mathrm{x} / \mathrm{b} ! \mathrm{E}, \mathrm{RD}>$ is called a register definition statement. The register definition statement is described as an n-tuple of substitution statements $\left[\mathrm{SS}_{1}, \ldots, \mathrm{SS}_{\mathrm{n}}\right]$ where each $\mathrm{SS}_{\mathrm{j}}$ must be $" R_{j} \leftarrow E_{j}\left(x, R_{1}, \ldots, R_{n}\right) "$. Here, " $E_{j}\left(x, R_{1}, \ldots, R_{n}\right) "$ must be a P-term which may contain the input variable " $x$ " and register variables " $R_{1},, \ldots,{ }_{n}$ ". In the labeled directed graph representing the EFSM, the substitution statement " $R_{j} \leftarrow R_{j}$ " is omitted. Note that the values of registers are changed after the output is emitted, that is, the value of the output is calculated based on the current register values.

We assume that there is a reset event for each state in a given EFSM. That is, we assume that

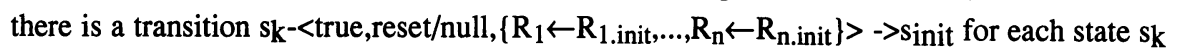
where sinit denotes the initial state of the EFSM and $R_{j . i n i t}$ denotes the initial value of the resister $\mathbf{R}_{\mathbf{j}}$. Such reset events are not described explicitly in the labeled directed graph representing the EFSM. The I/O event "reset/null" may be abbreviated as "reset". And we assume that the EFSM is completely specified. That is, for each pair of a state and an input, a corresponding transition must be specified. In the labeled directed graph representing the EFSM, a self-loop transition is omitted if its output is "null" and all the registers' values in the transition are not changed. We also assume that each state is reachable from the initial state. Hence, for any state $s_{k}$, there exists a transfer sequence from the initial state sinit to the state $\mathbf{s k}_{\mathbf{k}}$ (here, we may treat a sequence of $\mathrm{I} / \mathrm{O}$ events as a transfer sequence). Note that it is undecidable in general whether, for a given EFSM M and its state $\mathbf{s}_{\mathbf{k}}$, there exists a transfer sequence for leading $\mathbf{M}$ to trace from the initial state to the state $\mathbf{s}_{\mathbf{k}}$ because our EFSM/pres model can simulate Turing machines. However, if it exists, we can find it using a similar way to our test case derivation method which we propose in Section 4.1. For example, "a?0/e!0, a?1/e!0" is a transfer sequence from the initial state $s 1$ to the state $s 3$ in Fig. 1. Since we assume the reset events for all states, this assumption means that the EFSM is strongly connected.

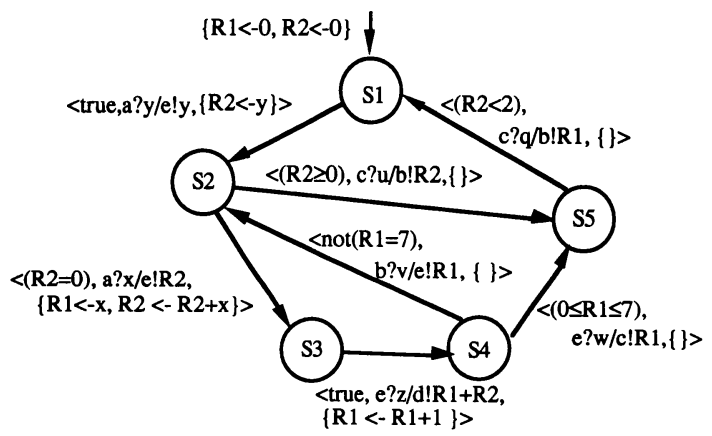

Fig. 1 An Example of Our EFSM/pres Model 
[Example 2.1]

Fig. 1 is an example of our EFSM/pres model. There are five states and two registers $R_{1}$ and $R_{2}$. The state $s_{1}$ is the initial state. The initial values $R_{1 \text {.init }}$ and $R_{2}$.init are both 0 . Suppose that the current state is $s_{2}$ and the current values of the registers $R_{1}$ and $R_{2}$ are both 0 . Then, since the transition condition $\left(R_{2}=0\right)$ at $s_{2} \rightarrow s_{3}$ is true at this case, the transition $s_{2} \rightarrow s_{3}$ is executable. If the input event " $a$ ? $x$ " is executed, then the current value " 0 " of the register $R_{2}$ is emitted to the gate "e". After the output is emitted, the input value " $x$ " is substituted to the register $R_{1}$. And the input value " $x$ " is added to the register $R_{2}$.

Although only the integer type is treated in our EFSM/pres model, we can also treat enumeration types, such as for instance, PDUtype $=\{C R, C C, D R, D C, D T\}$. Such enumeration types are very common in most specifications. The values of an enumeration type may be represented as integer values, and storing those values and branching by conditions using >, =, ,+- , etc. are sufficient to describe such protocols. Therefore, our EFSM/pres model has a considerable power for the purpose of system description. Many communication protocols such as OSI protocols can be described in EFSM/pres model. We give a specification in EFSM/pres model in Section 5. Also the specifications in EFSM/pres model can be easily described in other formal description languages such as SDL [CCITT 88], Estelle [ISO 89b] and LOTOS [ISO $89 \mathrm{a}]$.

\section{Application of General UIO Methods for EFSM's and Their Problems}

Some methods have been proposed for generating UIO sequences for FSM models (for survey, [BoUy 91]). In general, such methods cannot be applied for EFSM models. In this section, first, we explain the reason.

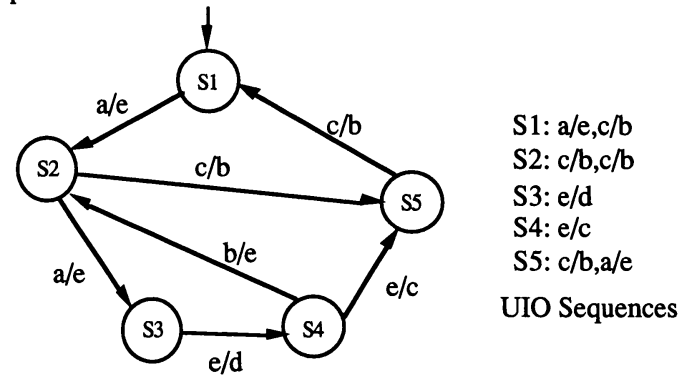

Fig. 2 FSM Transformed From EFSM in Fig. 1

Fig. 2 is the FSM which is obtained from the EFSM in Fig. 1 by ignoring the register values and I/O data. For the FSM, we can generate a UIO sequence for identifying each state (see Fig. 2) under the completeness assumption. Here, such UIO sequences are also treated as the UIO sequences for our EFSM/pres model. For example, "e/c" is a UIO sequence for identifying the state s4. The input/output "e/c" corresponds to the transition s4 $\rightarrow \mathrm{s} 5$. Let us consider how to apply the UIO sequence for the EFSM in Fig. 1. In Fig. 1, in order to execute the I/O event 
"e? $\mathrm{x} / \mathrm{c} ! \mathrm{R}_{1}$ " of the transition $\mathrm{s}_{4} \rightarrow \mathrm{s} 5$, the current value of the register $\mathrm{R}_{1}$ must satisfy its transition condition " $\left(0 \leq R_{1} \leq 7\right)$ ". However, this value is determined, for example, when the transition $s_{2} \rightarrow s_{3}\left(<\left(R_{2}=0\right), a ? x / e ! R_{2},\left\{R_{1} \leftarrow x, R_{2} \leftarrow R_{2}+x\right\}\right)$ is executed since the input value " $x$ " is substituted to the register $R_{1}$. And at the transition $s_{3} \rightarrow s_{4}$, the value of the register $R_{1}$ is incremented by one. Therefore, in order to execute the transition $s_{4} \rightarrow s_{5}$, the value " $x$ " satisfying the condition " $(0 \leq x+1 \leq 7)$ " must be input when the transition s2 $\rightarrow$ s3 (I/O event $\left.a ? x / e ! R_{2}\right)$ is executed. In order to execute the transition $s_{2} \rightarrow s_{3}$, the transition condition " $\left(R_{2}=0\right)$ " must hold. The value of $R_{2}$ is determined when the transition $s_{1} \rightarrow s_{2}(<$ true,a?y/e!y, $\left\{R_{2} \leftarrow y\right\}>$ ) is executed where the input value "y" is substituted to $R_{2}$. This input value "y" must be zero in order to execute the transition $s_{2} \rightarrow s_{3}$. That is, if we consider data values, then the event sequence $a ? y / e ! y, a ? x / e ! R_{2}, e ? z / d ! R_{1}{ }^{\prime}+R_{2}$ ', e? $w / c ! R_{1}$ " needs to be executed and the condition $\left\{\left(0 \leq x+1=R_{1}{ }^{\prime}+1=R_{1}{ }^{\prime \prime} \leq 7\right)\right.$ and $\left.\left(y=R_{2}=0\right)\right\}$ for the input data must hold. The event sequence corresponds to the transition sequence "s1 $\rightarrow s_{2} \rightarrow s_{3} \rightarrow s_{4} \rightarrow s_{5}$ ". Note that we use different register variables such as $R_{1}$ ' and $R_{1}$ " because those registers' values may be changed by executing each transition. From the above relations, the event sequence "a?y/e!y, a?x/e!y, $e ? z / d ! x+(y+x), e ? w / c ! x+1 "$ satisfying the condition " $(0 \leq x+1 \leq 7)$ and $(y=0) "$ must be executed. For example, since $\langle x, y, z, w\rangle=\langle 1,0,0,3\rangle$ is a solution satisfying the condition, the event sequence "a?0/e!0, a?1/e!0, e?0/d!2, e?3/c!2" is a test case with data values to execute the UIO sequence "s4 $\rightarrow \mathrm{s5}$ " (I/O event "e?x/c!R 1 ") described above.

In this paper, we call the transition sequence "s1 $\rightarrow \mathrm{s}_{2} \rightarrow \mathrm{s}_{3} \rightarrow \mathrm{s}_{4} \rightarrow \mathrm{s}_{5}$ " an extended UIO (E-UIO) sequence for identifying the state s4. The condition " $(0 \leq \mathrm{x}+1 \leq 7)$ and $(\mathrm{y}=0)$ " for the input variables is called the executability condition for the E-UIO sequence. We also call the event sequence "a?0/e!0, a?1/e!0, e?0/d!2, e?3/c!2" an extended UIO (E-UIO) sequence with data values for identifying the state s4. Hereafter, "e?3/c!2" is called a UIO sequence with data values. And the transition sequence "s1 $\rightarrow s_{2} \rightarrow s_{3} \rightarrow s_{4}$ " (the event sequence "a?0/e!0, $\mathrm{a}$ ?1/e!0, e?0/d!2") is called a preceding UIO sequence (with data values) for the UIO sequence "s4 $\rightarrow$ s5" ("e?0/c!1"). Each E-UIO sequence (with data values) consists of a pair of a preceding UIO sequence (with data values) and a UIO sequence (with data values). Note that the preceding UIO sequence may be null.

From the above discussion, we must find not only a UIO sequence with data values but also its preceding UIO sequence with data values in order to identify each state in a given EFSM. This is a difference for test case generations between FSM models and EFSM models. This problem has been also discussed in [UrYa 91, WaLi 92a, WaLi 92b].

\section{Test Case Generation for Our EFSM Model}

\subsection{Construction of E-UIO Sequences}

In this section, first, we define the E-UIO sequences formally. Here, we assume the number of input variables of each transition is one for simplicity of discussion. However, the properties presented in this section also hold for the case that the number of the input variables is more than one. 


$$
\mathrm{tr}_{-} \mathrm{t}, 0 \quad \text { tr_t }, 1 \quad \text { tr_t,h-1 }
$$

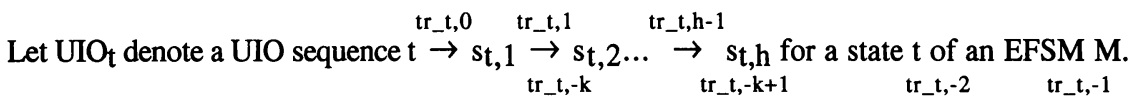

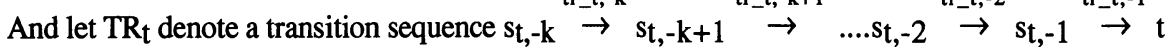

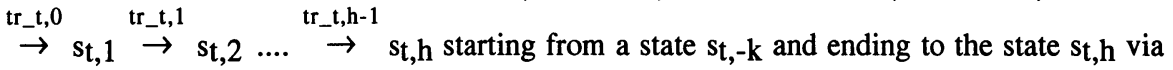
states $s_{t},-k+1, \ldots, s_{t},-2, s_{t},-1, t, s_{t}, 1, \ldots, s_{t, h}-1$ where $T_{t}$ contains the $U_{I O}$ as a postfix sequence. Here, we assume that each transition tr_t,i in $T R_{t}$ is "st,i- $<C_{i}, a_{i}$ ? $x_{i} / b_{i} ! E_{i}, R D_{i}>->s t, i+1$ "

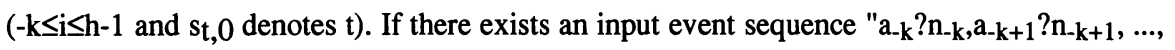

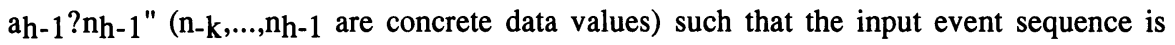
executable for any given registers' values $\mathrm{u}_{1}, \ldots, \mathrm{u}_{\mathrm{n}}$ at the state $\mathrm{st}_{\mathrm{t}, \mathrm{k}}$ of $\mathrm{M}$, then we say that the transition sequence $\mathrm{TR}_{\mathrm{t}}$ is an $E$-UIO sequence for the state $\mathrm{t}$ which corresponds to the given UIO sequence $\mathrm{UIO}_{\mathrm{t}}$. The sequence $\mathrm{st}_{\mathrm{t},-\mathrm{k}} \stackrel{\mathrm{tr}_{-} \mathrm{t}_{\mathrm{t}}-\mathrm{k}}{\rightarrow} \mathrm{st},-\mathrm{k}+1 \stackrel{\mathrm{tr}_{-} \mathrm{t}_{,}-\mathrm{k}+1}{\rightarrow} \ldots \mathrm{st},-2 \stackrel{\mathrm{tr}_{-} \mathrm{t}_{-}-2}{\rightarrow} \mathrm{st},-1 \stackrel{\mathrm{tr}_{-} \mathrm{t}_{-}-1}{\rightarrow} \mathrm{t}$ is called a preceding sequence of $\mathrm{UIO}_{t}$ and it is described as Pre(TR $\mathrm{TR}_{\mathrm{t}}$ ). For those input values and registers' values at the starting state $\mathrm{s}_{\mathrm{t}, \mathrm{k}} \mathrm{k}$, the corresponding output values are determined uniquely. We also say that the transition sequence with data values $\mathrm{TC}_{\mathrm{t}}=" \mathrm{a}_{-\mathrm{k}}$ ? $\mathrm{n}_{-\mathrm{k}} / \mathrm{b}_{-\mathrm{k}} ! \mathrm{v}_{-\mathrm{k}}, \ldots$,

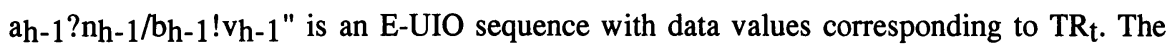
preceding sequence of $\mathrm{UIO}_{\mathrm{t}}$ with data values is described as $\operatorname{Pre}\left(\mathrm{TC}_{\mathrm{t}}\right)$. Formally, we define the E-UIO sequences as follows.

\section{[Definition 4.1]}

We say that a transition sequence TR $t$ of an EFSM M is an E-UIO sequence for a state $t$ of $M$ if and only if both the conditions (1) and (2) in Step 3 of the following Procedure 4.1 hold.

\section{Procedure 4.1}

Input : a transition sequence $\mathrm{TR}_{\mathrm{t}}=\mathrm{st},-\mathrm{k} \stackrel{\mathrm{tr}-\mathrm{t},-\mathrm{k}}{\rightarrow} \mathrm{st},-\mathrm{k}+1 \stackrel{\mathrm{tr}_{-} \mathrm{t},-\mathrm{k}+1}{\rightarrow} \cdots . . \mathrm{st},-2 \stackrel{\mathrm{tr}_{-} \mathrm{t},-2}{\rightarrow} \mathrm{st}_{,-1} \stackrel{\mathrm{tr}_{-} \mathrm{t},-1}{\rightarrow} \mathrm{t} \stackrel{\mathrm{tr}+\mathrm{t}, 0}{\rightarrow}$ tr_t, 1

$s_{t, 1} \stackrel{-}{\rightarrow} s t, 2 \ldots .$.

Variables : integer variable $p$.

variables $\mathrm{CR}_{\mathbf{j}}(\mathrm{j}=1, \ldots, \mathrm{n})$ for storing P-terms.

variables $\Phi$ TRt and $C^{\prime}$ for storing P-sentences.

$1 . p \leftarrow-\mathrm{k} . \mathrm{CR}_{\mathrm{j}} \leftarrow " \mathrm{R}_{\mathrm{j}}$ " for $\mathrm{j}=1, \ldots, \mathrm{n} . \quad \Phi \mathrm{TRt} \leftarrow$ true. $\mathrm{C}^{\prime} \leftarrow$ true.

2. While $p<h$, repeat the following four steps.

2.1 Calculate a P-sentence $C^{\prime}$ which is obtained from $C_{p}$ by replacing every $R_{j}$ and $x p$ in $\mathrm{C}_{p}$ with $\mathrm{CR}_{\mathrm{j}}$ and $\mathrm{y} p(\mathrm{j}=1, \ldots, \mathrm{n})$, respectively, where "y $p$ " is a new variable. ${ }^{1}$

$2.2 \Phi$ TRt $\leftarrow \Phi_{\mathrm{TRt}}$ and $\mathrm{C}^{\prime}$

2.3 Suppose that the substitution statement for each register $R_{j}(j=1, \ldots, n)$ in $R D_{p}$ is described as $" R_{j} \leftarrow E_{j}\left(x_{p}, R_{1}, \ldots, R_{n}\right) "$. Then, modify the contents of $C R_{1}, \ldots, C_{n}$ as follows :

$$
\mathrm{CR}_{1} \leftarrow \mathrm{E}_{1}\left(\mathrm{y}_{p}, \mathrm{CR}_{1}, \ldots, \mathrm{CR}_{\mathrm{n}}\right), \ldots, \mathrm{CR}_{\mathrm{n}} \leftarrow \mathrm{E}_{\mathrm{n}}\left(\mathrm{y}_{p}, \mathrm{CR}_{1}, \ldots, \mathrm{CR}_{\mathrm{n}}\right) .
$$

Those substitutions must be executed simultaneously.

$2.4 p \leftarrow p+1$

1 Since there is a case that $x_{i}$ of $\operatorname{tr} \_t, i$ and $x_{j}$ of $\operatorname{tr} \_t, j(i \neq j)$ use the same variable name in a given EFSM, we use a different new variable name " $y_{p}$ " for each input variable " $x_{p}$ " of $t_{-}, t, p$. 
3. If (1) TRt contains a UIO sequence $\mathrm{UIO}_{t}$ for a state $t$ of the EFSM $M$ as a postfix sequence and if (2) $\exists x_{-k}, \ldots, x_{h}-1 \forall R_{1}, \ldots, R_{n}[\Phi T R t]$ is true, i.e., there exist concrete data values for the input variables $\mathrm{x}_{-\mathrm{k}}, \ldots, \mathrm{xh}_{\mathrm{h}} 1$ such that the value of $\Phi_{\mathrm{TRt}}$ is true regardless of the register values $R_{1}, \ldots, R_{n}$ at the state $s t,-k$, then we say that $T R_{t}$ is an E-UIO sequence for the state $t$. If one of these conditions does not hold, then $\mathrm{TR}_{t}$ is not an E-UIO sequence.

Here, we say that $\Phi_{T R t}$ is the executability condition for the transition sequence $T_{t}$. The executability condition $\Phi_{T R_{t}}$ denotes the condition which the EFSM must satisfy to execute the given transition sequence $\mathrm{TR}_{\mathrm{t}}$. For example, if the transition sequence $\mathrm{TR}_{\mathrm{s}_{4}}=\mathrm{s}_{1} \rightarrow \mathrm{s}_{2} \rightarrow \mathrm{s}_{3} \rightarrow$ $s 4 \rightarrow s 5 "$ in Fig. 1 is given, the executability condition $\Phi_{T R s 4}$ is " $(0 \leq x+1 \leq 7)$ and $(y=0) "$. Since $\exists \mathrm{x}, \mathrm{y}, \mathrm{z}, \mathrm{w} \forall \mathrm{R}_{1}, \mathrm{R}_{2}\left[\Phi_{\mathrm{TR}} \mathrm{R}_{\mathrm{s}}\right]$ is true, $\mathrm{TR}_{\mathrm{S} 4}$ is an E-UIO sequence for the state s4. The values of the input variables not contained in $\Phi_{T R} 4$ can be arbitrarily determined. The output data values are determined depending on those input values and register values at the starting state of $\Phi_{\mathrm{TR}} \mathrm{s}_{4}$. However, for the transition sequence TR's4="s3 $\rightarrow \mathrm{s}_{4} \rightarrow \mathrm{s}^{\prime}$ ", the executability condition $\Phi_{T R}{ }^{\prime} 4$ is " $\left(0 \leq R_{1}+1 \leq 7\right) "$ and $\exists z, w \forall R_{1}, R_{2}\left[\Phi_{T R}{ }^{\prime} 4\right]$ is false. Therefore, TR's4 is not an E-UIO sequence for the state $s 4$.

Note that we call a given transition sequence as an E-UIO sequence if and only if an E-UIO sequence with data values can be generated for any register values at the starting state of the transition sequence. Therefore, we do not treat a transition sequence as an E-UIO sequence if the corresponding E-UIO sequence with data values can be generated only for some specific registers' values at the starting state. This condition is used when the E-UIO sequences are connected with their transfer sequences. For example, since we assume that each state of a given EFSM M is reachable from the initial state sinit, there is a transfer sequence $\alpha$ with data values leading the EFSM M to trace from the initial state sinit to the state st,-k. When the transfer sequence $\alpha$ is executed, the values of all registers are also determined. Using those values, an E-UIO sequence $\mathrm{TC}_{\mathrm{t}}$ with data values starting from the state $\mathrm{s}_{\mathrm{t}, \mathrm{k}} \mathrm{k}$ is obtained. By connecting the transfer sequence $\alpha$ and the E-UIO sequence $\mathrm{TC}_{\mathrm{t}}$ with data values, we can obtain a test case for checking the existence of the state $t$. In order to prove that each state of a given IUT (Implementation Under Test) is correctly implemented, for example, the UIOv-method in [VuCh 89] can be used where a test method for general EFSM models is described.

\section{[Lemma 4.1]}

For a given transition sequence $T_{t}$, the executability condition $\Phi_{T R t}$ is a P-sentence, which may contain some integer variables (input variables and register variables).

(Proof) From the definition in Section 2, all transition conditions are P-sentences. In Procedure 4.1, some P-term variables, say $\mathrm{CR}_{\mathrm{j}}$, may be replaced by $\mathrm{E}_{\mathrm{j}}\left(\mathrm{y}_{p}, \mathrm{CR}_{1}, \ldots, \mathrm{CR}_{\mathrm{n}}\right)$ based on the substitution statements such as " $R_{j} \leftarrow E_{j}\left(x_{p}, R_{1}, \ldots, R_{n}\right)$ " In Section 2 , we also assume that each $\mathrm{E}_{\mathrm{j}}\left(\mathrm{x}_{p}, \mathrm{R}_{1}, \ldots, \mathrm{R}_{\mathrm{n}}\right)$ must be a P-term. And $\mathrm{CR}_{1}, \ldots, \mathrm{CR}_{\mathrm{n}}$ are also P-terms. Therefore, the replaced expression $\mathrm{E}_{\mathrm{j}}\left(\mathrm{y}_{p}, \mathrm{CR}_{1}, \ldots, \mathrm{CR}_{\mathrm{n}}\right)$ is also $\mathrm{P}$-sentences. So, all $\mathrm{C}_{-}, \ldots, \mathrm{C}_{h-1}$ are $\mathrm{P}$ sentences even if $R_{\mathbf{j}}$ in each $C p$ is replaced with $C_{\mathbf{j}}$. Then, this lemma holds.

A logical expression containing only $\exists, \forall$ and P-sentences is called a Presburger sentence [HoUl 79]. It is known to be decidable whether a given Presburger sentence is true or not [HoUl 79]. 
[Property 4.1]

For any executability condition $\Phi_{\mathrm{TR}}$, it is decidable whether the expression $\exists \mathrm{x}_{-\mathrm{k}}, \ldots, \mathrm{xh}_{\mathrm{h}-1}$ $\forall R_{1}, \ldots, R_{n}\left[\Phi T R_{t}\right]$ is true or not. And if it is true, a solution $\left\langle n_{-k}, \ldots, n_{h-1}>\right.$ for the input

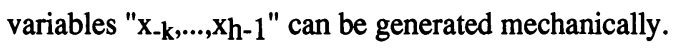

Since, in general, it takes much time to decide the truth of Presburger sentences whose forms are $\exists x_{-k}, \ldots, x_{h}-1 \forall R_{1}, \ldots, R_{n}\left[\Phi_{T R}\right]$, the following property is useful to reduce the computation time for generating the E-UIO sequences with data values.

[Property 4.2 (A Sufficient Condition)]

If a given executability condition $\Phi_{T R t}$ does not contain register variables $R_{1}, \ldots, R_{n}$ and if $\exists \mathrm{x}_{-\mathrm{k}}, \ldots, \mathrm{xh}_{\mathrm{h}-1}\left[\Phi_{\mathrm{TR}}\right]$ is true, then $\exists \mathrm{x}_{-\mathrm{k}}, \ldots, \mathrm{xh}_{\mathrm{h}-1} \forall \mathrm{R}_{1}, \ldots, \mathrm{R}_{\mathrm{n}}\left[\Phi_{\mathrm{TR}}\right]$ is also true.

From this property, in Step 3 of Procedure 4.1, if (1) $\Phi_{T R_{t}}$ does not contain register variables, (2) TR $t$ contains a UIO sequence for a state $t$ as a postfix sequence, and (3) $\exists x_{-k}, \ldots$. , $\mathrm{xh}_{\mathrm{h}-1}\left[\Phi_{\mathrm{TR}}\right]$ is true, then $\mathrm{TR}_{\mathrm{t}}$ is an E-UIO sequence for the state $\mathrm{t}$. Under this sufficient condition, a solution satisfying $\Phi_{\mathrm{TR}}$ can be generated through integer linear programming [HiBo 94]. One way to generate a solution is as follows. By replacing "not $\left(E_{1} \geq E_{2}\right)$ " into " $\left(E_{1}<E_{2}\right)$ ", and by replacing "not $\left(E_{1}=E_{2}\right)$ " into " $\left(E_{1}<E_{2}\right)$ or $\left(E_{1}>E_{2}\right)$ " and so on, we can transform a given $\Phi_{T R}$ into an equivalent $\mathrm{P}$-sentence $\phi^{\prime}\left(\mathrm{x}_{-\mathrm{k}}, \ldots, \mathrm{xh}_{\mathrm{h}}-1\right)$ which does not contain "not" operators. Let

$$
\phi_{1}{ }^{\prime}\left(x_{-k}, \ldots, x_{h-1}\right) \text { or } \phi_{2}{ }^{\prime}\left(x_{-k}, \ldots, x_{h}-1\right) \text { or } \ldots \text { or } \phi_{m}{ }^{\prime}\left(x_{-k}, \ldots, x_{h}-1\right)
$$

be a disjunctive normal form of $\phi^{\prime}\left(x_{-k}, \ldots, x_{h-1}\right)$ where each $\phi_{q}{ }^{\prime}\left(x_{-k}, \ldots, x_{h-1}\right)(1 \leq q \leq m)$ is a conjunction of some linear inequalities. So, $\Phi_{T R_{t}}$ is satisfiable if and only if, for some $q$ $(1 \leq \mathrm{q} \leq \mathrm{m}), \phi_{\mathrm{q}}{ }^{\prime}\left(\mathrm{x}_{-\mathrm{k}}, \ldots, \mathrm{xh-1}\right)$ is satisfiable. By regarding each linear inequality in $\phi_{\mathrm{q}}{ }^{\prime}\left(\mathrm{x}_{-\mathrm{k}}, \ldots, \mathrm{xh}_{\mathrm{h}-1}\right)$ as a constraint on an integer linear programming problem, we can decide whether the integer linear programming problem has integer solutions. And if it has a solution, a procedure solving integer linear programming problems can mechanically generate a solution $\left\langle\mathrm{n}_{-\mathrm{k}}, . ., \mathrm{n}_{\mathrm{h}-1}\right\rangle$ satisfying $\Phi_{\text {TRt }}$.

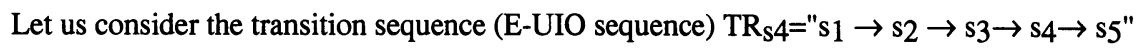
in Fig. 1 again. For this transition sequence TR $\mathbf{S}_{4}, \Phi_{\mathrm{TR}} 4$ is $"(0 \leq x+1 \leq 7)$ and $(y=0) "$. Since $\Phi_{\mathrm{TR} s 4}$ does not contain register variables and $\exists \mathrm{x}, \mathrm{y}, \mathrm{z}, \mathrm{w}\left[\Phi_{\mathrm{TR} s}\right]$ is true, this means that $\exists x, y, z, w \forall R_{1}, R_{2}\left[\Phi_{T R}\right.$ ] $]$ is also true. And a solution, say $\langle x, y, z, w>=<1,0,0,3>$, is obtained mechanically. Since the variables "z" and "w" are not appeared in $\Phi_{T R}$, any values may be given as their values. Using those values, we can generate an E-UIO sequence "a?0/e!0, $\mathrm{a} ? 1 / \mathrm{e} ! 0, \mathrm{e} ? 0 / \mathrm{d} ! 2, \mathrm{e} ? 3 / \mathrm{c} ! 2^{\prime \prime}$ with data values for identifying the state s4. Since the starting state of the E-UIO is the initial state $s 1$, we can check whether the state $s 4$ is correctly implemented in a given IUT by giving the input event sequence of this E-UIO from the initial state and observing its output event sequence.

We can not determine the values of outputs directly from $\Phi_{T R t}$ if the output values depend on the registers' values at the starting state of $\mathrm{TR}_{\mathrm{t}}$. For example, let us generate an E-UIO sequence for identifying the state s3 in Fig. 1. Let $\mathrm{TR}_{\mathrm{s}} 3$ denote the transition "s3 $\rightarrow$ s4". TR $\mathbf{s} 3$ is a UIO sequence for the state $\mathrm{s} 3$. By giving $\mathrm{TR}_{\mathrm{S} 3}$ to Procedure 4.1 , the executability condition $\Phi_{\mathrm{TR} \text { 3 } 3}$ is "true". Since $\mathrm{TR}_{\mathrm{S} 3}$ satisfies the conditions in Procedure $4.1, \mathrm{TR}_{\mathrm{s} 3}$ is also an E-UIO 
sequence for the state $\mathrm{s} 3$. In this case, any integer value, say "1", may be given as the input value " $z$ ". However, the output value " $R_{1}+R_{2}$ " is determined depending on the transfer sequence which has been executed before executing this E-UIO sequence. For example, in order to identify the state s3, we must lead a given IUT to trace the initial state $s 1$ to the state s3 and then we must give the E-UIO sequence TR 3 . There exists a transfer sequence, for example "a?0/e!0, a?1/e!0", for leading the EFSM to trace the initial state $s 1$ to the state s3. If the transfer sequence "a?0/e!0, a?1/e!0" is executed from the initial state, then the registers $\mathrm{R}_{1}$ and $\mathrm{R}_{2}$ store 1 and 1 , respectively. Then, the value of " $R_{1}+R_{2}$ " is " 2 " when the EFSM enters the state 33 . That is, "e?1/d!2" is an E-UIO sequence with data values for identifying the state s3. By connecting "a?0/e!0, a?1/e!0" and "e?1/d!2", we can check whether the state $s 3$ is correctly implemented in a given IUT.

In both cases, we can generate the transition sequences with data values satisfying the executability condition $\Phi_{T R t}$ mechanically if all registers' values at the starting state of $T_{t}$ are given.

For the EFSM in Fig. 1, E-UIO sequences are given in Fig. 3. Let E-UIO sk denote the EUIO sequence for identifying the state $s \mathrm{k}$ in Fig. 3. Integer " 0 " is given as the values of the registers "R1" in E-UIO 2 and $\mathrm{E}_{1} \mathrm{UIO}_{\mathrm{S} 5}$ since the initial register value of "R $\mathrm{R}_{1}$ " at the initial state $s_{1}$ is " 0 ". In E-UIO 3 , integer "2" is given as the value of " $R_{1}+R_{2}$ " since the value of " $\mathrm{R}_{1}+\mathrm{R}_{2}$ " becomes "2" when the EFSM enters the state $\mathrm{s} 3$ after executing a transfer sequence "a?0/e!0, a?1/e!0" from the initial state.

\begin{tabular}{|c|c|c|c|c|}
\hline State & I/O event seq. & $\Phi_{\text {TRsk }}$ & \multicolumn{2}{|c|}{$\mathrm{TR}_{\mathrm{sk}}=\mathrm{E}-\mathrm{UIO}_{\mathrm{sk}} \mathrm{TC}_{\mathrm{sk}}=\mathrm{E}-\mathrm{UIO}$ with data values } \\
\hline s1 & $\mathrm{a} ? \mathrm{y} / \mathrm{e} ! \mathrm{y}, \mathrm{c} ? \mathrm{u} / \mathrm{b} ! \mathrm{y}$ & $(y \geq 0)$ & $\mathrm{s}_{1} \rightarrow \mathrm{s}_{2} \rightarrow \mathrm{s}_{5}$ & $\mathrm{a} ? 1 / \mathrm{e} ! 1, \mathrm{c} ? 4 / \mathrm{b} ! 1$ \\
\hline s2 & $\begin{array}{l}\text { a?y/e!y,c?u/b!y, } \\
\mathrm{c} ? \mathrm{q} / \mathrm{b} ! \mathrm{R}_{1}\end{array}$ & $\begin{array}{l}(y \geq 0) \\
\text { and }(y<2)\end{array}$ & $\mathrm{s}_{1} \rightarrow \mathrm{s}_{2} \rightarrow \mathrm{s}_{5} \rightarrow \mathrm{s}_{1}$ & $\begin{array}{l}\mathrm{a} ? 1 / \mathrm{e} ! 1, \mathrm{c} ? 4 / \mathrm{b} ! 1 \\
\mathrm{c} ? 3 / \mathrm{b} ! 0\end{array}$ \\
\hline s3 & $\mathrm{e} ? \mathrm{z} / \mathrm{d} ! \mathrm{R}_{1}+\mathrm{R}_{2}$ & true & s3 $\rightarrow$ s4 & $\mathrm{e} ? 1 / \mathrm{d} ! 2$ \\
\hline s4 $\mathrm{e} ? \mathrm{z}$ & $\begin{array}{c}\text { a?y/e!y, a?x/e!y, } \\
z / d ! x+(y+x), e ? w / c ! x+1\end{array}$ & $\begin{array}{r}(0 \leq x+1 \leq 7) \\
\text { and }(y=0)\end{array}$ & $\begin{aligned} \mathrm{s}_{1} & \rightarrow \mathrm{s}_{2} \rightarrow \mathrm{s}_{3} \\
& \rightarrow \mathrm{s}_{4} \rightarrow \mathrm{s}_{5}\end{aligned}$ & $\begin{array}{l}\text { a?0/e!0, a?1/e! } 0 \\
\text { e?0/d!2, e?3/c!2 }\end{array}$ \\
\hline s5 & $\begin{array}{l}\text { a?y'/e!y',c?u/b!y', } \\
\text { c?q/b!R } 1, a ? y / e ! y\end{array}$ & $\begin{array}{l}\left(y^{\prime} \geq 0\right) \\
\text { and }\left(y^{\prime}<2\right)\end{array}$ & $\begin{aligned} \mathrm{s}_{1} & \rightarrow \mathrm{s}_{2} \rightarrow \mathrm{s}_{5} \\
& \rightarrow \mathrm{s}_{1} \rightarrow \mathrm{s}_{2}\end{aligned}$ & $\begin{array}{l}\text { a?1/e!1,c?4/b!1, } \\
\mathrm{c} ? 3 / \mathrm{b} ! 0, \mathrm{a} ? 0 / \mathrm{e} ! 0\end{array}$ \\
\hline
\end{tabular}

Note that each $R_{i}$ in $\mathrm{I} / \mathrm{O}$ event seq. denotes the value of register $R_{i}$ at the starting state of TRsk.

Fig. 3 E-UIO Sequences

\section{[Theorem 4.1]}

For a given UIO sequence $\mathrm{UIO}_{t}$ for identifying a state $t$ of an EFSM $M$, if there exists an $\mathrm{E}$ UIO sequence with data values containing $\mathrm{UIO}_{t}$ as a postfix sequence, then we can generate it mechanically.

(Proof) Using breadth first search, we can enumerate every transition sequence $\mathrm{TR}_{\mathrm{t}}$ containing $\mathrm{UIO}_{\mathrm{t}}$ as a postfix sequence. If $(1) \exists \mathrm{x}_{-\mathrm{k}}, \ldots, \mathrm{xh}_{\mathrm{h}-1} \forall \mathrm{R}_{1}, \ldots, \mathrm{R}_{\mathrm{n}}\left[\Phi \mathrm{TR}_{\mathrm{t}}\right]$ is true, then $\mathrm{TR}_{t}$ is an $\mathrm{E}$ UIO sequence. From Property 4.1 , it is decidable whether (1) is true or not. And if it is satisfiable, then concrete data values for $\mathrm{TR}_{t}$ is obtained from a solution satisfying $\Phi_{T R t}$ and the 
registers' values at the starting state of $\mathrm{TR}_{\mathrm{t}}$ mechanically. If there exists an E-UIO sequence, such an E-UIO sequence can be found by breadth first search eventually.

Let $\mathrm{st}_{\mathrm{t}, \mathrm{k}} \mathrm{k}$ denote the starting state of $\mathrm{TR}_{\mathrm{t}}$. And let $\alpha$ denote a transfer sequence leading $\mathrm{M}$ from the initial state to the state st,-k. We apply Steps 1 and 2 of Procedure 4.1 for this $\alpha$. If the condition ( $\left.1^{\prime}\right) \exists x_{-k-j}, . ., x_{-k-1} \forall R_{1}, . ., R_{n}\left[\left\{R_{1}=R_{1 . i n i t}\right.\right.$ and .. and $\left.\left.R_{n}=R_{n . i n i t}\right\} \supset \Phi_{\alpha}\right]$ is true (here,

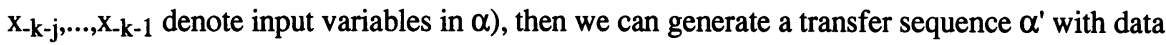
values leading $M$ from the initial state to the state $s t,-k$ mechanically. Since, in this paper, we assume that all states of an EFSM M are reachable from the initial state, such a sequence $\alpha^{\prime}$ can be eventually generated by enumerating possible transition sequences leading $M$ from the initial state to the state st,-k.

Since our EFSM/pres model can simulate Turing machines (two counter automata), it is undecidable whether there exists an E-UIO sequence with data values for a given UIO sequence. There may exist a case that there does not exist an E-UIO sequence with data values for a given UIO sequence. For such a case, the breadth first search for enumerating possible transition sequences may continue forever. Therefore, we must decide by ourselves when we stop the enumeration.

\subsection{Test Cases for Checking Correctness of Transitions}

Next, we will explain how to generate test cases for checking correctness of transitions. Let $\mathrm{UIO}_{\mathrm{V}}$ and $\mathrm{UIO}_{\mathrm{W}}$ denote UIO sequences for identifying given states $\mathrm{v}$ and $\mathrm{w}$ of an EFSM M, respectively. Suppose that there is a transition $v-<C, a$ ? $x / b ! E, R D>->w(v \rightarrow w)$. Let $\beta$ be a transition sequence starting from a state $t$ and entering to the state $v$. Now, suppose that $\beta$ is the preceding sequence $\mathrm{Pre}\left(\mathrm{E}-\mathrm{UIO} \mathrm{V}_{\mathrm{v}}\right)$ of an E-UIO sequence $\mathrm{E}-\mathrm{UIO}_{\mathrm{v}}$ corresponding to $\mathrm{UIO}_{\mathrm{V}}$ and that $" \beta, v \rightarrow w "$ is the preceding sequence Pre(E-UIO $\left.{ }_{W}\right)$ of an E-UIO sequence E-UIO corresponding to $\mathrm{UIO}_{\mathrm{W}}$. Let $\beta_{\mathrm{V}}$ denote the preceding sequence Pre(TC(E-UIO $\left.\mathrm{V}_{\mathrm{V}}\right)$ ) of an E-UIO sequence TC(E-UIO $\left.)_{v}\right)$ with data values and let " $\beta_{w}^{\prime}, a n_{a} / b ! n_{b}$ " denote the preceding sequence $\operatorname{Pre}\left(\mathrm{TC}\left(\mathrm{E}-\mathrm{UIO}_{\mathrm{W}}\right)\right)$ of an E-UIO sequence TC(E-UIO $\left.{ }_{\mathrm{W}}\right)$ with data values. If there exist E-UIOs $\mathrm{TC}\left(\mathrm{E}-\mathrm{UIO}_{\mathrm{v}}\right)$ and $\mathrm{TC}\left(\mathrm{E}-\mathrm{UIO}_{\mathrm{W}}\right)$ with data values such that $\beta_{\mathrm{v}}$ and $\beta_{\mathrm{w}}^{\prime}$ are the same, then we can check the correctness of transitions as follows.

Let $\alpha^{\prime}$ be a transfer sequence with data values leading $M$ from the initial state to the state $t$. Then, by executing $\alpha^{\prime}$ and TC(E-UIO $\left.{ }_{\mathrm{V}}\right)$ sequentially, we can check whether the IUT enters the state $t$ after $\alpha^{\prime}$ and $\beta_{\mathrm{v}}$ are executed. Therefore we can select " $\alpha^{\prime}, \beta_{\mathrm{v}}$ " as a transfer sequence with data values leading $M$ from the initial state to the state $v$ and execute $\alpha^{\prime}$ and TC(E-UIO $\left.\mathrm{W}_{\mathrm{W}}\right)$ sequentially. Since the heading sequence " $\alpha^{\prime}, \beta^{\prime}{ }^{\prime}$ " of $\operatorname{TC}\left(E-U I O O_{w}\right)$ is the same as " $\alpha$ ', $\beta_{v}$ ", the IUT enters the state $v$ after " $\alpha$ ', $\beta^{\prime}{ }_{w}$ " is executed. At the state $v$, by checking the $I / O$ event $\mathrm{a} ? \mathrm{n}_{\mathrm{a}} / \mathrm{b}$ ! $_{\mathrm{b}}$, we can check whether the transition $\mathrm{v} \rightarrow \mathrm{w}$ " is correctly implemented. By executing the rest of TC(E-UIO $)$, we can check whether the IUT enters the state $w$ after the transition " $\mathrm{v} \rightarrow \mathrm{w}$ " is executed.

Here, we say that the pair of E-UIO $\mathrm{V}$ and $\mathrm{E}-\mathrm{UIO}_{\mathrm{W}}$ satisfying the above conditions is a pair of test cases for checking correctness of the transition $\mathrm{v} \rightarrow \mathrm{w}$ ". We also say that the pair of 
$\mathrm{TC}\left(\mathrm{E}-\mathrm{UIO}_{\mathrm{V}}\right)$ and $\mathrm{TC}\left(\mathrm{E}-\mathrm{UIO}_{\mathrm{W}}\right)$ is a pair of test cases for checking correctness of the transition "v $\rightarrow$ w" with data values.

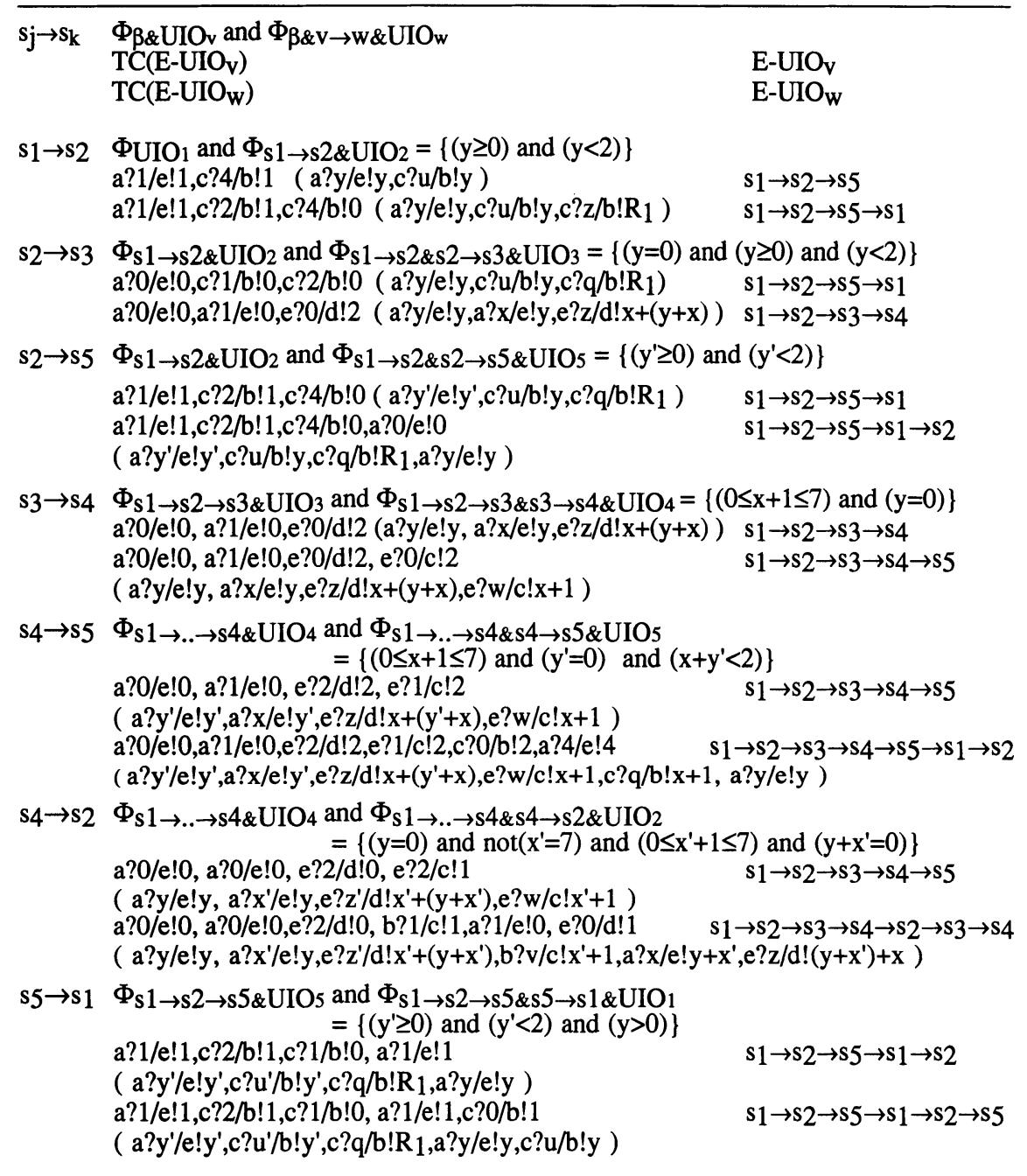

Fig. 4 Pairs of Test Cases for Checking Correctness of Transitions

For the EFSM in Fig. 1, we can generate the pairs of test cases for checking correctness of all transitions. In Fig. 4, for each transition "sj $\rightarrow s k_{k}$ ", the pair of E-UIO ${ }_{s j}$ and E-UIO $s k$ is shown. The pair of TC(E-UIO $\left.{ }_{s j}\right)$ and TC(E-UIO transition "s2 $\rightarrow$ s3", first, the executability condition $\Phi$ UIOs $2="(R 2 \geq 0)$ and $(R 2<2)$ " for a UIO

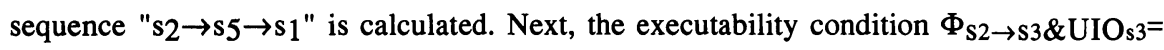
"(R2=0)" for the transition "s2 $\rightarrow$ s3" followed by a UIO sequence "s3 $\rightarrow$ s4" for the state $s 3$ is calculated( $\mathrm{t}_{1} \& \mathrm{t}_{2}$ denotes a sequence $\mathrm{t}_{1}$ followed by $\left.\mathrm{t}_{2}\right)$. In order to execute both "s $\mathrm{s}_{2} \rightarrow \mathrm{s}_{5} \rightarrow \mathrm{s}_{1}$ " and "s2 $\rightarrow s_{3} \rightarrow s_{4}$ ", the conjunction of two executability conditions $\Phi$ UIOs2 anc 
$\Phi_{\mathrm{S} 2 \rightarrow \mathrm{s} 3 \& U I O s 3}$ must hold. However, since the conjunction contains the register variable "R2" and it is not true for some register value, we append the preceding transition "s1 $\rightarrow s_{2}$ " and modify the executability conditions. Then, the new conditions $\Phi_{\mathrm{S} 1 \rightarrow \mathrm{s} 2 \& U \mathrm{UIO}_{\mathrm{s} 2}="(\mathrm{y}=0) " \text { and }}$

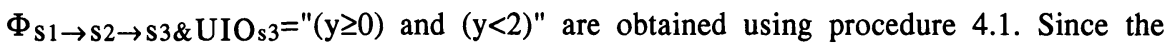

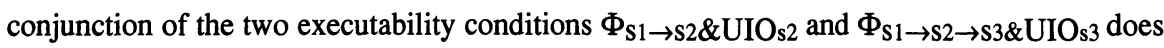
not contain register variables and it is satisfiable, we can obtain the pair $<\mathrm{TC}\left(\mathrm{E}-\mathrm{UIO}_{\mathrm{S} 2}\right), \mathrm{TC}(\mathrm{E}-$ $\mathrm{UIO}_{\mathrm{s} 3}$ ) > of test cases with data values for checking correctness of the transition "s $2 \rightarrow \mathrm{s} 3$ ".

Note that, in Fig. 4, in order to generate a pair of test cases for checking correctness of the transition $\mathrm{s}_{4} \rightarrow \mathrm{s}_{2}$, we used another UIO sequence $\mathrm{s}_{2} \rightarrow \mathrm{s}_{3} \rightarrow \mathrm{s}_{4}$ as $\mathrm{UIO}_{\mathrm{s} 2}$.

[Theorem 4.2]

We can generate a pair of test cases with data values for checking correctness of a transition $\mathrm{v}-<\mathrm{C}, \mathrm{a}$ ? $\mathrm{x} / \mathrm{b}$ !E,RD $>->\mathrm{w}(\mathrm{v} \rightarrow \mathrm{w})$ of an EFSM M mechanically if it exists.

(Proof) We can enumerate every transition sequence $\beta$ entering the state $\mathrm{v}$ using breadth first search and construct $\Phi_{\beta \& U I O}$ and $\Phi_{\beta \& v \rightarrow \text { w\&UIO }}$ mechanically from given $\mathrm{UIO}_{\mathrm{v}}, \mathrm{UIO}_{\mathrm{W}}$ and the transition $\mathrm{v} \rightarrow \mathrm{w}$. Since the truth of $\exists \mathrm{x}_{-\mathrm{k}}, \ldots, \mathrm{xh}_{\mathrm{h}}-1, \ldots \forall \mathrm{R}_{1}, \ldots, \mathrm{R}_{\mathrm{n}}\left[\Phi_{\beta \& U I O}\right.$ and $\left.\Phi_{\beta \& \mathrm{~V} \rightarrow \mathrm{w} \& \mathrm{UIO}}\right]$ is also decidable, the theorem holds.

\section{Example of EFSM Model and its Test Case Generation}

In this section, we give a practical example of our EFSM model and generate test cases for the example. A simplified specification for a part of the OSI Session protocol [ISO 87] is shown in Fig. 6 where the gates and registers in Fig. 5 are used.

The specification is extracted from the data transfer phase of kernel, duplex, minor synchronization, major synchronization and resynchronization functional units of the OSI Session protocol. The protocol machine has four gates (see Fig. 5). The gate C (Command) is an input gate from the upper layer. The gate I (Indication) is an output gate to the upper layer. The gate $S$ (Send) is an output gate to the lower layer. The gate $R$ (Receive) is an input gate from the lower layer. We assume the reset events for all states. Here, we gave the following simplifications :

(i) omitting minor synchronization acknowledgment (MIA) PDU and register V(A);

(ii) assuming that the protocol machine possesses the major token and minor token and never transfers those ; and

(iii) choosing only restart option among three options for resynchronization.

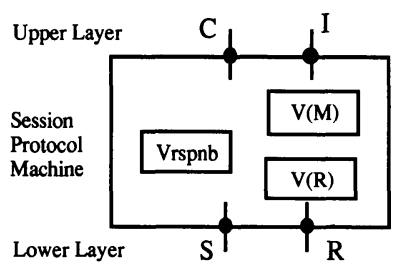

Fig. 5 Gates and Registers 


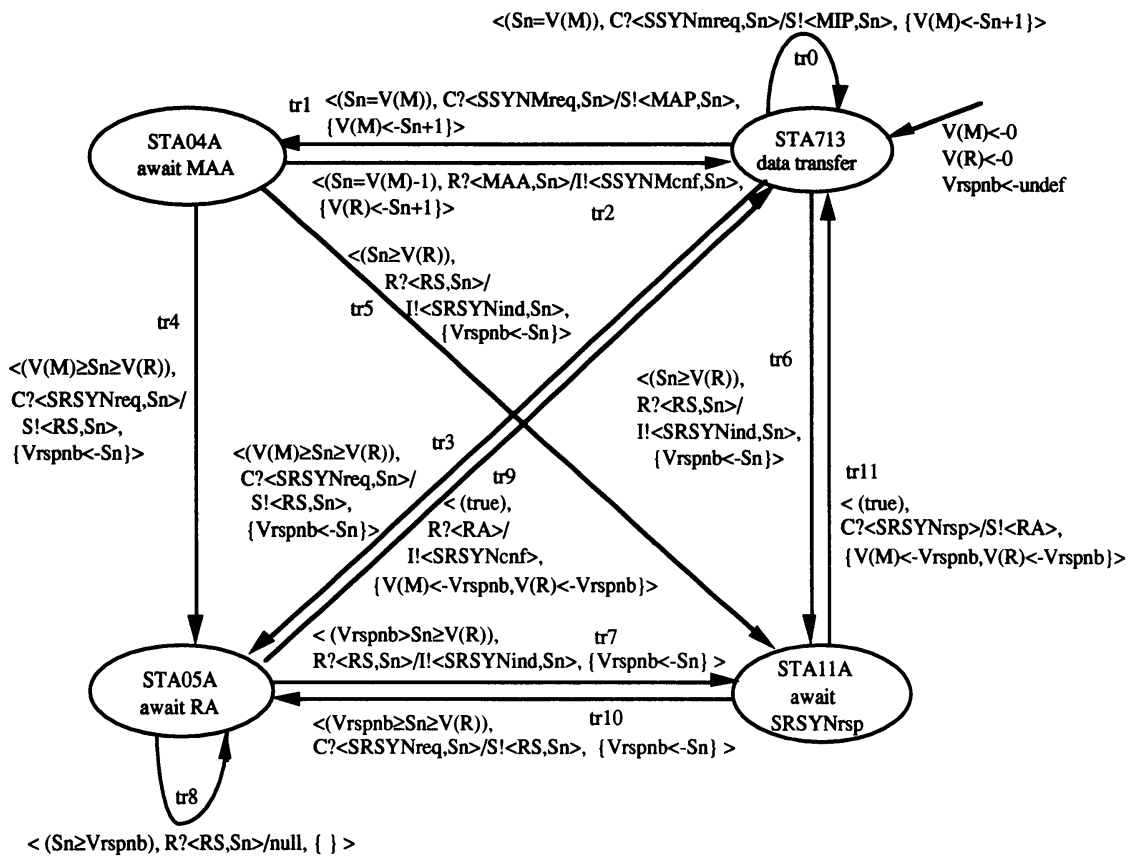

Fig. 6 Example Specification of Simplified Session Protocol

The protocol uses serial numbers for synchronization. Every command or PDU contains a serial number. The register $\mathrm{V}(\mathrm{M})$ stores the next serial number to be used. The register $\mathrm{V}(\mathrm{R})$ stores the lowest serial number to which resynchronization is permitted. The register $V_{\text {rspnb }}$ stores the lowest serial number of requested resynchronizations. At the state STA713, the protocol machine accepts the minor and major synchronization request (SSYNmreq and SSYNMreq) commands from the upper layer. At the state STA04A, the machine awaits the major synchronization acknowledgment (MAA) PDU from the lower layer. The resynchronization starts from the state STA713 or STA04A by accepting the resynchronization request (SRSYNreq) command from the upper layer or receiving the resynchronization (RS) PDU from the lower layer. At the state STA05A, the protocol machine awaits the resynchronization acknowledgment (RA) PDU or a stronger RS PDU from the lower layer. At the state STA11A, the machine awaits the resynchronization response (SRSYNrsp) command or a stronger SRSYNreq command from the upper layer.

By treating the enumeration types such as the commands and PDU's as some integer constants, the specification in Fig. 6 can be treated as our EFSM/pres model. Here, we treat the pair of an I/O gate name and its command as the input/output when we generate the E-UIO sequences. For example, at the state STA713, there is a transition "C? $<$ SSYNmreq,Sn $>$ / $\mathrm{S} !<\mathrm{MIP}, \mathrm{Sn}>$ " (STA713 $\rightarrow$ STA713). Here, we treat the pairs $<$ C,SSYNmreq $>$ and $<$ S,MIP $>$ of gate names and commands as the input and output of this transition when we generate the $\mathrm{E}$ - 
UIO sequences. In Fig. 7, E-UIO sequences with data values for identifying each state in Fig. 6 are shown. The pairs of test cases for checking correctness of all transitions can be also derived mechanically.

\begin{tabular}{|c|c|c|c|}
\hline State & $\begin{array}{l}\text { E-UIO } \\
\text { ( E-UIO with data values ) }\end{array}$ & Condition & Transition \\
\hline STA713 & $\begin{array}{l}\text { reset,C? }<\text { SSYNmreq,Sn }>/ \mathrm{S} !<\mathrm{MIP}, \mathrm{Sn}> \\
(\text { reset,C? }<\mathrm{SSYNmreq}, 0>/ \mathrm{S} !<\mathrm{MIP}, 0>\text { ) }\end{array}$ & $(\mathrm{Sn}=0)$ & STA713 $\rightarrow$ STA713 \\
\hline STA04A & $\begin{array}{c}\text { reset,C?<SSYNMreq,S'n }>/ S !<M A P, S^{\prime} n>\text {, } \\
\text { R? }<\text { MAA,Sn }>/ I !<\text { SSYMcnf,Sn }> \\
\text { ( reset,C?<SSYNMreq,0>/S!<MAP,0>, } \\
\text { R? }<\text { MAA, } 0>/ I !<S S Y M c n f, 0>\text { ) }\end{array}$ & $\left(S^{\prime} n=0\right)$ and $\left(S n=\left(S^{\prime} n+1\right)-1\right)$ & $\begin{array}{l}\text { STA713 } \rightarrow \text { STA04A } \\
\rightarrow \text { STA713 }\end{array}$ \\
\hline STA05A & $\begin{array}{l}\mathrm{R} ?<\mathrm{RA}>/ I !<\mathrm{SRSYNcnf}> \\
(\mathrm{R} ?<\mathrm{RA}>/ \mathrm{I} !<\mathrm{SRSYNcnf}>)\end{array}$ & true & STA05A $\rightarrow$ STA713 \\
\hline STA11A & $\begin{array}{l}\text { C? }<\text { SRSYNrsp }>/ S !<\text { RA }> \\
(\mathrm{C} ?<\text { SRSYNrsp }>/ S !<\text { RA }>)\end{array}$ & true & STA11A $\rightarrow$ STA713 \\
\hline
\end{tabular}

Fig. 7 E-UIO Sequences

\section{Conclusion}

In this paper, we have proposed a new technique to generate E-UIO sequences mechanically for a restricted class of EFSM's. We have been developing a tester for generating E-UIO sequences. In order to generate each E-UIO sequence, some integer linear programming problems must be solved. In our experiences, the transition conditions are very simple for most practical examples. Such an observation is also shown in [ChZh 94]. From those results, we can conclude that the number of the constraints of integer linear programming problems which we must solve is at most 10 or 20 in most cases. It takes about 20 second for our tester to solve the integer linear programming problems whose constraints' numbers are 30 using a SUN SPARCstation ELC (16MB Memory) in most cases. Since the E-UIO sequences may not be so long and then computation time is not so much, we believe our approach can be used for many practical examples. One of the future works is to show the usefulness of our approach using the tester.

\section{Acknowledgments}

The authors would like to thank Prof. A. Petrenko of Universite de Montréal, Canada and anonymous reviewers for their useful comments for improving the paper.

\section{References}

[BoUy 91] B. S. Bosik and M. U. Uyar : "Finite State Machine Based Formal Methods in Protocol Conformance Testing", Computer Networks ISDN Systems, 22, pp. 733, 1991.

[CCITT 88] CCITT : "SDL : Specification and Description Language", Recommendation Z.100, Nov. 1988. 
[ChZh 94] S. T. Chanson and J. Zhu : "Automatic Protocol Test Suite Derivation", Prof. IEEE INFOCOM'94, pp.792-799, 1994.

[EeWo 92] H. Eertink and D. Wolz : "Symbolic Execution of LOTOS Specifications", Proc. 5th Int. Conf. on Formal Description Techniques (FORTE'92), pp.295-310, North-Holland, Oct. 1992.

[HiBo 92] T. Higashino, G. v. Bochmann, X. Li, K. Yasumoto and K. Taniguchi : "Test System for a Restricted Class of LOTOS Expressions with Data Parameters", Proc. 5th Int. Workshop on Protocol Test Systems (IWPTS'92), pp.205-216, North-Holland, Sept. 1992.

[HiBo 94] T. Higashino and G. v. Bochmann : "Automatic Analysis and Test Case Derivation for a Restricted Class of LOTOS Expressions with Data Parameters", IEEE Trans. on Software Engineering, Vol. 20, No. 1, pp. 29-42, Jan. 1994.

[HoU1 79] J. E. Hopcroft and J. D. Ullman : "Introduction to Automata Theory, Languages, and Computation", Addison-Weslay, 1979.

[ISO 89a] ISO : "Information Processing System, Open Systems Interconnection, LOTOS A Formal Description Technique Based on the Temporal Ordering of Observational Behaviour", IS 8807, Jan. 1989.

[ISO 87] ISO : "Information Processing System - Open Systems Interconnection - Basic Connection Oriented Session Protocol Specification", IS 8327, Aug. 1987.

[ISO 89b] ISO : "Estelle : A Formal Description Technique Based on an Extended State Transition Model", ISO 9074, July 1989.

[Sari 87] B. Sarikaya, G. v. Bochmann and E. Cerny : "A Test Design Methodology for Protocol Testing", IEEE Trans. on Soft. Eng., pp. 518-531, May 1987.

[TrSa 89] P. Tripathy and B. Sarikaya : "Test Generation from Protocol Specification", Proc. 2nd Int. Conf. on Formal Description Techniques (FORTE'89), pp.329343, North-Holland, Nov. 1989.

[Tret 89] J. Tretmans : "Test Case Derivation from LOTOS Specifications", Proc. 2nd Int. Conf. on Formal Description Techniques (FORTE'89), pp.345-359, NorthHolland, Nov. 1989.

[UrYa 91] H. Ural and B. Yang : "A Test Sequence Selection Method for Protocol Testing", IEEE Transactions on Communications, Vol. 39, No. 4, pp.514-523, April 1991.

[VuCh 89] S. T. Vuong, W.L. Chan and M. R. Ito : "The UIOv-Method for Protocol Test Sequence Generation", Proc. 2nd Int. Workshop on Protocol Test Systems, pp.161-175, North- Holland, Oct. 1989.

[WaLi 92a] C.-J. Wang and M. T. Liu : "Axiomatic Test Sequence Generation for Extended Finite State Machines", Proc. of the 12th Int. Conf. on Distributed Computing Systems (ICDCS-12), pp. 252-259, June 1992.

[WaLi 92b] C.-J. Wang and M. T. Liu : "A Test Suite Generation method for Extended Finite State Machines using Axiomatic Semantics Approach", Proc. of the 12th IFIP Symp. on Protocol Specification, Testing and Verification (PSTV-XII), pp. 2943, North-Holland, June 1992. 